\title{
OPEN Adaptive value of foot-and-mouth disease virus capsid substitutions with opposite effects on particle acid stability
}

Flavia Caridi ${ }^{1}$, Rodrigo Cañas-Arranz $^{1}$, Ángela Vázquez-Calvo ${ }^{1}$, Patricia de León ${ }^{1}$,
Katherine I. Calderón ${ }^{1}$, Esteban Domingo ${ }^{1}$, Francisco Sobrino ${ }^{1 \bowtie}$ \& Miguel A. Martín-Acebes

Foot-and-mouth disease virus (FMDV) is a picornavirus that exhibits an extremely acid sensitive capsid. This acid lability is directly related to its mechanism of uncoating triggered by acidification inside cellular endosomes. Using a collection of FMDV mutants we have systematically analyzed the relationship between acid stability and the requirement for acidic endosomes using ammonium chloride $\left(\mathrm{NH}_{4} \mathrm{Cl}\right)$, an inhibitor of endosome acidification. A FMDV mutant carrying two substitutions with opposite effects on acid-stability (VP3 A116V that reduces acid stability, and VP1 N17D that increases acid stability) displayed a rapid shift towards acid lability that resulted in increased resistance to $\mathrm{NH}_{4} \mathrm{Cl}$ as well as to concanamicyn $\mathrm{A}$, a different lysosomotropic agent. This resistance could be explained by a higher ability of the mutant populations to produce $\mathrm{NH}_{4} \mathrm{Cl}$-resistant variants, as supported by their tendency to accumulate mutations related to $\mathrm{NH}_{4} \mathrm{Cl}$-resistance that was higher than that of the WT populations. Competition experiments also indicated that the combination of both amino acid substitutions promoted an increase of viral fitness that likely contributed to $\mathrm{NH}_{4} \mathrm{Cl}$ resistance. This study provides novel evidences supporting that the combination of mutations in a viral capsid can result in compensatory effects that lead to fitness gain, and facilitate space to an inhibitor of acid-dependent uncoating. Thus, although drug-resistant variants usually exhibit a reduction in viral fitness, our results indicate that compensatory mutations that restore this reduction in fitness can promote emergence of resistance mutants.

Whether a trade-off exists between capsid stability and evolvability is an important issue for viral evolution. Viral capsids protect the genetic material from environmental insult to warrant functional integrity of the viral genome $^{1}$. However, capsids are not static containers as they are also subjected to multiple selective pressures that include immune responses, receptor recognition, physicochemical factors or antiviral drugs. Accordingly, capsid proteins are under continuous evolution leading, among others, to extensive antigenic variation, generation of drug-resistant mutants or adaptation to unfavorable environmental conditions. This evolutionary potential is constrained by the need for a balance between functionality and stability because capsids have to be stable enough to protect the genetic material while permitting its release within target host cells.

Picornaviruses are simple models to study the evolution of viral capsids. Its icosahedral capsid is formed by 60 copies of each of the four structural proteins (VP1-VP4) arranged into 12 pentameric subunits ${ }^{2}$. Interestingly, picornavirus capsids differ remarkably in their sensitivity to acidic $\mathrm{pH}$, ranging from the resistance of Enterovirus to the extreme acid-lability of the Aphthovirus foot-and-mouth disease virus (FMDV) ${ }^{3}$. FMDV is the etiological agent of a highly devastating disease of cloven-hoofed animals that affects important livestock species such as swine, cattle, goats or sheep ${ }^{4}$. As described for other viruses, the acid-lability of FMDV capsid has been related to its mechanism of uncoating inside cellular endosomes ${ }^{5,6}$. The current model supports that acidic $\mathrm{pH}$ inside endosomes triggers FMDV capsid dissociation into pentameric subunits ${ }^{7}$. This may be produced by electrostatic repulsions originated from the protonation of key histidine residues at $\mathrm{pHs}$ slightly below neutrality ${ }^{8}$. Thus, neutralization of endosomal $\mathrm{pH}$ using ammonium chloride $\left(\mathrm{NH}_{4} \mathrm{Cl}\right)$, -which acts as proton sink within endosomes impairing their acidification-, inhibited FMDV infection by preventing virus uncoating ${ }^{9-12}$. Accordingly, the

${ }^{1}$ Centro de Biología Molecular "Severo Ochoa" (CSIC-UAM), 28049 Madrid, Spain. ${ }^{2}$ Department of Biotechnology, Instituto Nacional de Investigación y Tecnología Agraria y Alimentaria (INIA, CSIC), 28040 Madrid, Spain. "email: fsobrino@cbm.csic.es 
relation between acid-dependent uncoating and sensitivity to $\mathrm{NH}_{4} \mathrm{Cl}$ has provided an interesting model for the study of the effects of a host-targeting antiviral on viral evolution ${ }^{11,13}$.

The quasispecies organization of FMDV implies that viral populations are complex and dynamic distributions of variants with a huge potential for variation ${ }^{14}$. This has enabled the isolation from FMDV mutant distributions of a broad panel of mutant FMDVs differing in their sensitivity to acidic $\mathrm{pH}$, shedding light on the molecular mechanisms of FMDV uncoating ${ }^{12,13,15-20}$. Remarkably, the capsid of FMDV displays a high degree of plasticity to tolerate mutations, and provides a good model system for the study of the genetic mechanisms that contribute to viral evolution ${ }^{21-28}$. Mutations affecting capsid stability have provided evidence for multifunctional residues ${ }^{18}$, the existence of additive effects ${ }^{13,18,29}$, or the accommodation of compensatory mutations to restore viral fitness ${ }^{25,30,31}$, supporting that the stability of FMDV capsid is a multifactorial trait due to the interaction between residues from different capsid proteins ${ }^{13}$.

In this work, we have analyzed the relationship between acidic $\mathrm{pH}$ sensitivity and resistance to inhibition of endosome acidification using $\mathrm{NH}_{4} \mathrm{Cl}$. Our results show that the combination of two amino acid substitutions with opposite effects on acid-stability resulted in an increase in viral fitness. Such an increase mediated the adaptation to the inhibition of endosomal acidification exerted by $\mathrm{NH}_{4} \mathrm{Cl}$ through rapid selection of resistant variants.

\section{Materials and methods}

Viruses, infections and titrations. All the FMDV variants were recovered from their respective infectious cDNA clones derived from pMT28 plasmid, which contains the complete genomic sequence of the type C FMDV isolate C-S8c1 (wild type, WT) ${ }^{32}$. The viruses used in this study were the WT FMDV, the single mutants VP1 V11I, VP1 N17D, VP2 H145Y, VP3 A116V, and VP3 A118V; the double mutants VP2 H145Y + VP1 N17D, VP3 A116V + VP1 N17D, and VP1 N17D + VP1 T12A; and the triple mutants VP3 A116V + VP1N17D + VP1 T12A, and VP3 A116T + VP3 A118V + VP1 N17D. The origin of the mutations and the procedures followed for infectious cDNA clone manipulation, in vitro transcription and RNA-transfection have been previously described $^{11-13,18}$. To minimize effects of viral amplification on the mutant spectrum composition, viruses were directly harvested at $48 \mathrm{~h}$ after transfection of BHK-21 cells with the corresponding in vitro synthesized viral RNA. The identities of the consensus sequence of the viral populations recovered from transfections were verified by nucleotide sequencing ${ }^{11}$. Unless otherwise specified, viral stocks were produced by transfection of in vitro synthesized RNA and two serial passages of the virus recovered. Procedures for infections in liquid medium and virus titration in semisolid agar medium were as described ${ }^{10,11}$. Virus titer was calculated as the number of plaque-forming units (PFU)/mL by standard plaque assay using semisolid agarose medium. The multiplicity of infection (MOI) defined as the number of PFU/cell in each experiment is indicated in the corresponding figure legend.

Acid-inactivation assays. Procedures for determination of acid-sensitivity of FMDV have been previously described ${ }^{11,33}$. Briefly, equal number of PFU from each population were treated with PBS buffer adjusted to different $\mathrm{pHs}$ for $30 \mathrm{~min}$, then the buffer was neutralized and the remaining infectivity in each sample was determined by standard titration in BHK-21 cells.

Inhibitors and treatments. Inhibition of endosome acidification using $\mathrm{NH}_{4} \mathrm{Cl}$ (Merck, Darmstadt, Germany) was performed as described ${ }^{11}$. Cells were incubated in culture medium containing $25 \mathrm{mM} \mathrm{NH}_{4} \mathrm{Cl}$ (plus $25 \mathrm{mM}$ HEPES at $\mathrm{pH} 7.4$ to buffer extracellular $\mathrm{pH}$ ) from $1 \mathrm{~h}$ prior to infection and throughout the rest of the assay. For inhibition of endosome acidification using concanamycin A, cells were treated for 30 min before infection with $100 \mathrm{nM}$ concanamycin A (Sigma; St. Louis, MO) and the drug was maintained only during the first hour of infection ${ }^{11}$. Control cells were treated in parallel with drug vehicle (DMSO). Treatment with guanidinium hydrochloride ( $\mathrm{GuHCl}$; Sigma) was performed as described ${ }^{34}$. One hour prior to infection cells were incubated with $4 \mathrm{mM} \mathrm{GuHCl}$ that was maintained for the rest of the experiment.

Next-generation sequencing (NGS). Cells were infected in the presence or in the absence of $\mathrm{NH}_{4} \mathrm{Cl}$ (MOI of $0.01 \mathrm{PFU} /$ cell) in triplicate wells. Viral RNA was extracted from each sample and cDNA was synthesized. A fragment of 463 nucleotides in length comprised between the $\mathrm{C}$ terminus of VP3 and the N terminus of VP1 was amplified using oligonucleotide primers CGGGACAATCAACCTACAC GTTGGTTATCCGACACTG coupled to nucleotide adapters CS1/CS2. Library preparation, amplification and sequencing were performed at the Parque Científico de Madrid using a MiSeq equipment (Illumina, San Diego, CA, USA) using the $2 \times 300 \mathrm{bp}$ MiSeq Reagent Kit v3. A total of 6231577 were analyzed (average reads per sample 519,298 $\pm 122,251$ ).

Biological fitness assessment by competition experiments. Equal number of PFU of FMDV WT and FMDV with substitutions A116V in VP3 and N17D in VP1 (abbreviated VP3 A116V + VP1 N17D) populations were mixed (initial MOI of $0.1 \mathrm{PFU} /$ cell [0.05 for each virus]) and used to infect BHK-21 cells treated or not with $\mathrm{NH}_{4} \mathrm{Cl}$. Viruses recovered from these infections were harvested and further passaged 10 times under the same conditions. The nucleotide sequence of the capsid coding region was determined by cDNA synthesis and automated nucleotide sequencing as described ${ }^{12}$. The proportions of the competing genomes were estimated from the chromatograms as a ratio of the integrated areas of each nucleotide under each peak of mutated position $^{12,30}$.

Statistics. Data analysis was performed using GraphPad PRISM 7 for Windows (GraphPad Software Inc., San Diego, CA, USA). Two tailed Student's t test $P$ values between control and drug-treated samples were cor- 
a

\begin{tabular}{|c|c|c|c|c|c|}
\hline \multirow{2}{*}{ Virus } & \multicolumn{3}{|c|}{ Amino acid substitutions } & \multirow{2}{*}{$\begin{array}{l}\text { Uncoating } \mathrm{pH} \\
\left(\mathrm{pH}_{50}\right)\end{array}$} & \multirow{2}{*}{ Ref. } \\
\hline & VP3 & VP2 & VP1 & & \\
\hline - (WT) & & & & 6.65 & 13 \\
\hline$\square$ & & $\mathrm{H} 145 \mathrm{Y}$ & N17D & 5.35 & 18 \\
\hline$\Delta$ & & & N17D & 6.05 & 18 \\
\hline$\nabla$ & & $\mathrm{H} 145 \mathrm{Y}$ & & 6.3 & 18 \\
\hline$\nabla$ & A118V & & & 6.93 & 13 \\
\hline$\Delta$ & A116V & & & 6.87 & 13 \\
\hline $\mathbf{\square}$ & A116V & & N17D & 6.67 & 13 \\
\hline$x$ & A116V & & N17D T22N & 6.87 & 13 \\
\hline$\bullet$ & & & N17D T12A & 6.93 & 13 \\
\hline$\bullet$ & & & V11I & 6.92 & 13 \\
\hline+ & $\begin{array}{l}\text { A116T } \\
\text { A118V }\end{array}$ & & N17D & 6.90 & 13 \\
\hline
\end{tabular}
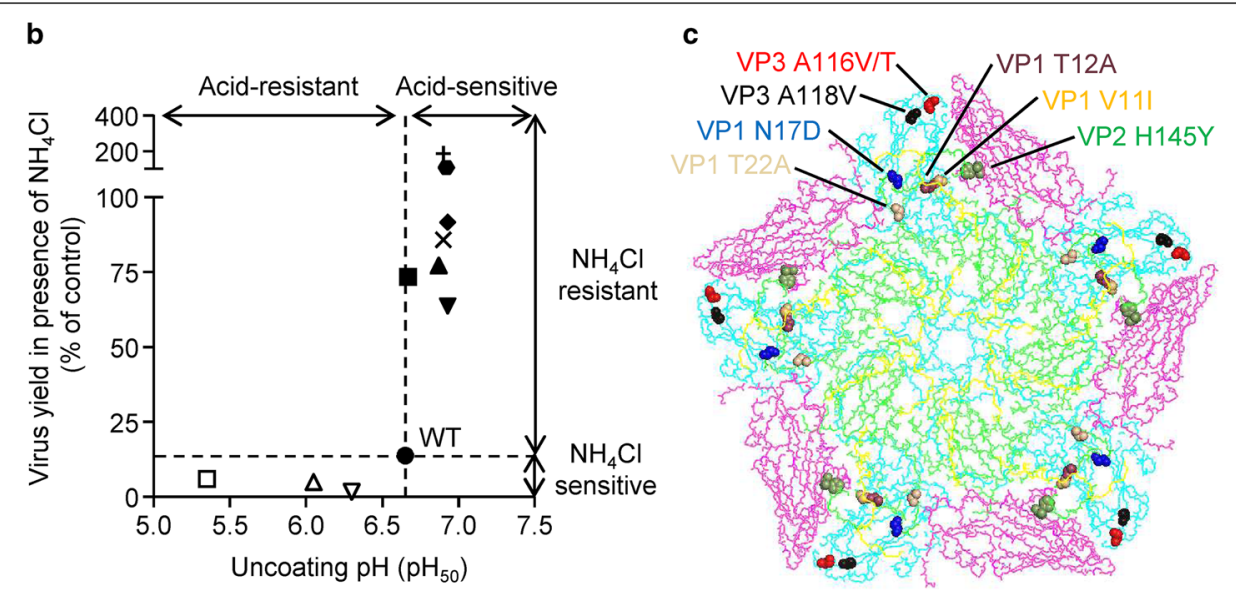

Figure 1. Double FMDV mutant with VP3 A116V and VP1 N17D does not follow the relation between uncoating $\mathrm{pH}$ and resistance to $\mathrm{NH}_{4} \mathrm{Cl}$ in FMDV. (a) Combinatorial mutants analyzed in the study. Symbols in the first column identify Each mutant, with the amino acid substitutions, the uncoating $\mathrm{pH}\left(\mathrm{pH}_{50}\right)$ and references included in the columns on the right. See text for details. (b) Relation between uncoating $\mathrm{pH}$ and sensitivity to endosomal neutralization by $\mathrm{NH}_{4} \mathrm{Cl}$ of FMDV mutants ${ }^{13,18}$. FMDV mutants are identified with the symbols explained in (a). BHK-21 cells treated or not with $25 \mathrm{mM} \mathrm{NH}_{4} \mathrm{Cl}$ were infected (MOI of $0.5 \mathrm{PFU} /$ cell) with FMDV C-S8c1 (WT) or its variants. Virus yield obtained in samples treated with $\mathrm{NH}_{4} \mathrm{Cl}$ was determined at $8 \mathrm{~h}$ post-infection, and is expressed as a percentage of that obtained in samples not treated with the drug. Mean virus yield $(n=3)$ of each mutant was plotted as a function of its uncoating $\mathrm{pH}$, estimated by $\mathrm{pH}_{50}$ value. (c) Location on the structure of FMDV C-S8c1 capsid ${ }^{44}$ of amino acid substitutions present in the mutants analyzed. An inside schematic view of a pentameric subunit is displayed. Only amino acid main chains are shown, for clarity. VP1 is green, VP2 is magenta, VP3 is cyan, and VP4 is yellow.

rected for multiple comparisons using the Sidak-Bonferroni method. Differences among viruses were analyzed by one-way analysis of the variance applying Bonferroni's correction for multiple comparisons. Asterisks in the figures denote $P$ values $\left({ }^{\star} P<0.05\right.$ and $\left.{ }^{* *} P<0.005\right)$. Unless otherwise specified, data represent the means \pm standard deviations (SDs). The number of independent experiments analyzed $(n)$ in each case is indicated in the corresponding figure legend.

\section{Results}

Double FMDV mutant VP3 A116V + VP1 N17D does not conform to the canonical relationship between uncoating $\mathrm{pH}$ and resistance to $\mathrm{NH}_{4} \mathrm{Cl}$. The uncoating of FMDV is triggered by acidification inside endosomes where viral particles are delivered following the endocytic route. A variety of studies support a correlation between acid-lability and resistance to the endosomal acidification blockage induced by $\mathrm{NH}_{4} \mathrm{Cl}^{7}$. Accordingly, FMDVs with increased acid lability display higher resistance to $\mathrm{NH}_{4} \mathrm{Cl}^{11,20}$. In contrast, mutants with increased acid stability display increased sensitivity to $\mathrm{NH}_{4} \mathrm{Cl}^{12,17,19}$.

To systematically test this hypothesis, we analyzed the sensitivity to $\mathrm{NH}_{4} \mathrm{Cl}$ of $10 \mathrm{FMDV}$ variants (Fig. 1A) differing in their uncoating $\mathrm{pH}$ (estimated by $\mathrm{pH}_{50}$, defined as the $\mathrm{pH}$ value that results in a $50 \%$ loss of infectivity) 


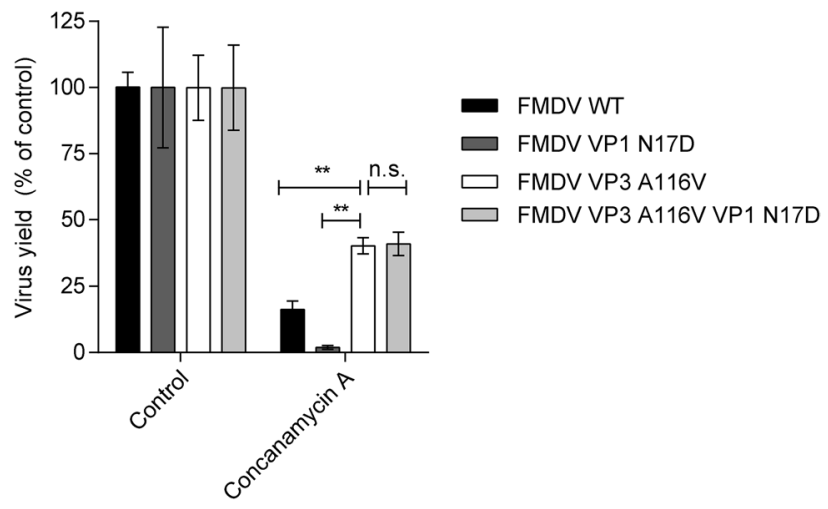

Figure 2. Double FMDV mutant VP3 A116V + VP1 N17D also displays increased resistance to the inhibitor of endosome acidification Concanamycin A. Analysis of the resistance of FMDV WT and FMDV VP3 A116V + VP1 N17D to Concanamycin A. BHK-21 cells treated or not with $100 \mathrm{nM}$ Concanamycin A, were infected with FMDV FMDV VP3 A116V VP1 N17D, single mutants VP3 A116V, VP1 N17D or WT at a MOI of $0.5 \mathrm{PFU} /$ cell. Virus yield at $8 \mathrm{~h}$ post-infection was determined by standard plaque assay. Two tailed Student's t-test $P$ values between double mutant and the rest of viruses were corrected for multiple comparisons using Bonferroni's method ${ }^{* \star} P<0.005$; n.s. non-significant). Data represent the means \pm SDs $(n=2-3)$.

(Fig. 1B). These viruses included wild type $\left(\mathrm{WT}, \mathrm{pH}_{50} 6.65\right)$, acid-resistant $\left(\mathrm{pH}_{50}<6.65\right)$ and acid-sensitive viruses $\left(\mathrm{pH}_{50}>6.65\right)$. The panel of viruses analyzed carried amino acid replacements in regions that have been proposed to modulate FMDV capsid stability through different mechanisms (Fig. 1C). These substitutions included: (1) bulkier residues in VP3 located close to the pentameric interface that destabilize inter pentameric interactions, destabilize viral capsid and increase its acid lability (A116V, A116T, and A118V) ${ }^{11,13}$; (2) amino acid replacements located in the N terminus of VP1 (N17D, T22N, T12A, and V11I) ${ }^{12,13}$ that regulate acid stability by a not well characterized mechanism in which only the relevance of the electrostatic interaction with negatively charged RNA has been identified for VP1 N17D mutant ${ }^{35}$; (3) the amino acid replacement VP2 H145Y located near the intra protomeric interface, region key for Aphthovirus stability ${ }^{36,37}$, which has been also shown to increase acid resistance in several FMDV serotypes ${ }^{17,18}$. As expected, the yield of acid-resistant viruses was more inhibited by $\mathrm{NH}_{4} \mathrm{Cl}$ than that of the WT. Acid-sensitive viruses were less susceptible or even fully resistant to the drug (Fig. 1B). Surprisingly, although its acid sensitivity $\left(\mathrm{pH}_{50}, 6.67\right)$ was similar to that of the WT, FMDV VP3 A116V + VP1 N17D showed a behavior very similar to that of acid-sensitive viruses, with increased resistance to $\mathrm{NH}_{4} \mathrm{Cl}$ in comparison to WT. Thus, this mutant deviates from the correlation between acid sensitivity and resistance to the inhibition of acid-dependent uncoating by means of endosomal acidification blockage with $\mathrm{NH}_{4} \mathrm{Cl}$.

Increased resistance of double FMDV mutant VP3 A116V + VP1 N17D against another inhibitor of endosomal acidification. The effect of Concanamycin A, an inhibitor of vacuolar-ATPase that blocks endosome acidification, was also analyzed on the infection of FMDV mutant VP3 A116V + VP1 N17D (Fig. 2). A significant increase in the resistance of FMDV mutant VP3 A116V + VP1 N17D against Concanamycin A was noticed when compared to WT and single mutant VP1 N17D, thus displaying a behavior similar to that of single mutant VP3 A116V. These results confirmed that the resistance of FMDV mutant VP3 A116V + VP1 N17D against endosome acidification applies also to inhibitors other than $\mathrm{NH}_{4} \mathrm{Cl}$.

Treatment with $\mathrm{NH}_{4} \mathrm{Cl}$ promotes a quick acid-lability shift of FMDV VP3 A116V+VP1 N17D. The virus carrying amino acid replacements A116V in VP3 and N17D in VP1 is a rationally designed double mutant that combined a determinant of acid sensitivity (VP3 A116V) and a determinant of acid resistance (VP1 N17D) in the same genome, yielding a virus with similar acid sensitivity than FMDV WT ${ }^{13}$. Searching for an explanation of the unexpected resistance of FMDV VP3 A116V + VP1 N17D to $\mathrm{NH}_{4} \mathrm{Cl}$ (Fig. 1b), we compared the inactivation profiles at different acidic $\mathrm{pHs}$ of this mutant, the corresponding single mutants, and FMDV WT, grown in the presence or in the absence of $\mathrm{NH}_{4} \mathrm{Cl}$. To this end, we analyzed the acid sensitivity of the infection progenies recovered at $8 \mathrm{~h}$ post-infection. Viruses were incubated in buffers adjusted to different $\mathrm{pH}$ values and the remaining infectious virus was determined by plaque assay (Fig. 3a-d). No significant changes in the acid inactivation profiles were noted in the presence of $\mathrm{NH}_{4} \mathrm{Cl}$ for FMDV WT or the single mutants (Fig. 3a-c). However, the $\mathrm{NH}_{4} \mathrm{Cl}$-treatment was sufficient to produce a significant decrease in the infectivity recovered upon incubation of FMDV VP3 A116V + VP1 N17D at a pH of 6.6, the uncoating pH of FMDV WT, when compared with progeny production in absence of $\mathrm{NH}_{4} \mathrm{Cl}$ (Fig. 3d). Thus, these results indicate that the augmented resistance to $\mathrm{NH}_{4} \mathrm{Cl}$ of FMDV VP3 A116V + VP1 N17D is concomitant with a rapid increase in acid lability of the viral population in response to $\mathrm{NH}_{4} \mathrm{Cl}$ treatment.

To analyze the genetic determinants of the altered $\mathrm{NH}_{4} \mathrm{Cl}$ sensitivity, the consensus sequence of the capsidcoding region of the $\mathrm{NH}_{4} \mathrm{Cl}$-treated population of FMDV VP3 A116V + VP1 N17D was determined. No changes in this sequence were observed suggesting that no additional amino acid replacements were responsible for this behavior. 
a

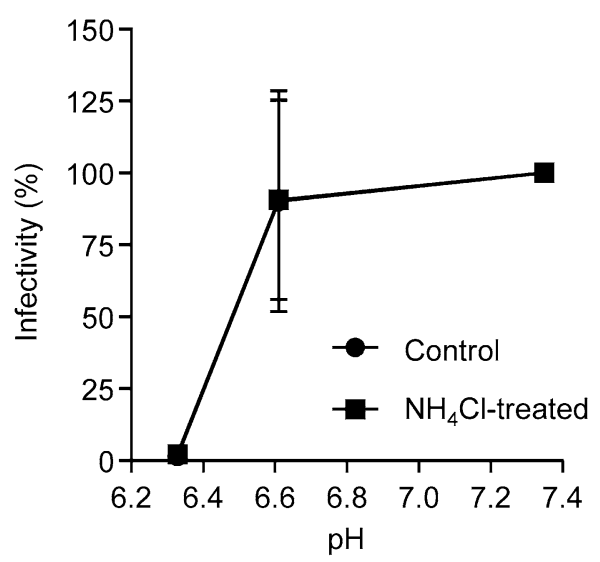

C

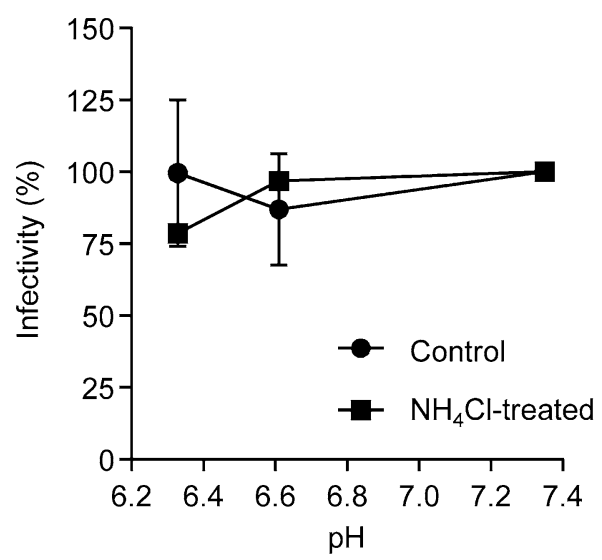

b

FMDV VP3 A116V

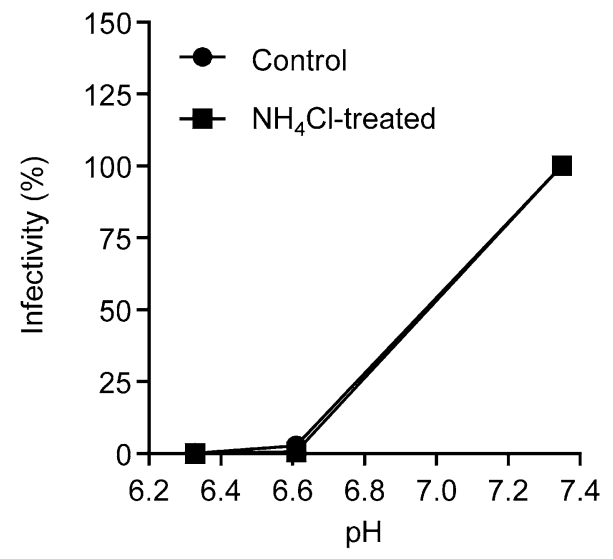

d

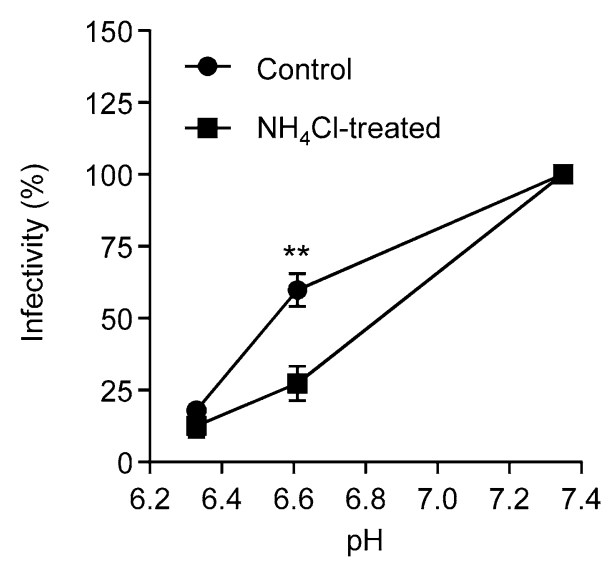

Figure 3. Acid-lability shift of FMDV VP3 A116V + VP1 N17D population after a single passage in the presence of $\mathrm{NH}_{4} \mathrm{Cl}$. Acid sensitivity profiles of WT (a), VP3 A116V (b), VP1 N17D (c), and double mutant VP3 A116V + VP1 N17D (d) after a single amplification in the presence of $\mathrm{NH}_{4} \mathrm{Cl}$. Viruses were grown in the presence or absence of $25 \mathrm{mM} \mathrm{NH}_{4} \mathrm{Cl}$ (MOI of $0.5 \mathrm{PFU} /$ cell) for $8 \mathrm{~h}$, and the acid sensitivity of the progeny populations was analyzed. For this purpose, equal number of PFU from each population were treated with PBS at $\mathrm{pH} 7.3,6.6$ and 6.3 for $30 \mathrm{~min}$ (abscissae). The $\mathrm{pH}$ was neutralized, and the remaining PFU in each sample was determined in BHK-21 cells. Infectivity was calculated as the percentage of PFU recovered at each different $\mathrm{pH}$ relative to that obtained at $\mathrm{pH} 7.3$ (ordinate). Two tailed Student's $t$-test $P$ values between control and $\mathrm{NH}_{4} \mathrm{Cl}$-treated populations were corrected for multiple comparisons using the Sidak-Bonferroni method $\left({ }^{*} P<0.005\right)$. Data represent the means \pm SDs $(n=3)$.

Increased frequency of $\mathrm{NH}_{4} \mathrm{Cl}$-resistant variants in FMDV VP3 A116V + VP1 N17D populations. To test if the increased resistance of FMDV VP3 A116V+VP1 N17D to $\mathrm{NH}_{4} \mathrm{Cl}$ applied also to other FMDV inhibitors that block infection by other mechanisms, we tested the effect of guanidine hydrochloride $(\mathrm{GuHCl})$, a compound that targets RNA dependent RNA polymerase and inhibits FMDV replication ${ }^{38,39}$. While FMDV VP3 A116V + VP1 N17D was less inhibited by $\mathrm{NH}_{4} \mathrm{Cl}$ than FMDV WT, GuHCl inhibited the infection of both viruses to a similar extent (Fig. 4a). These results indicated that resistance of FMDV VP3 A116V + VP1 $\mathrm{N} 17 \mathrm{D}$ to $\mathrm{NH}_{4} \mathrm{Cl}$ does not entail a general antiviral resistance.

Next, the frequency of $\mathrm{NH}_{4} \mathrm{Cl}$ and $\mathrm{GuHCl}$ resistant variants in FMDV VP3 A116V + VP1 N17D and FMDV WT populations was explored. To this end, the capacity to infect and produce lysis plaques in drug-treated monolayers was analyzed. In these experiments, viral populations directly recovered from transfection of in vitro synthesized viral RNA from the respective infectious cDNA clones, without further amplification, were used, hence minimizing the effects of viral amplification in quasiespecies variability. In the WT population, the percentage of $\mathrm{NH}_{4} \mathrm{Cl}$ resistant variants obtained (1.8\%) was in agreement with previous data ${ }^{11}$ (Fig. $4 \mathrm{~b}$ ). However, this percentage was significantly increased (32.7\%) in the case of FMDV VP3 A116 + VP1 N17D. In contrast, no significant differences between the resistant lysis plaques of the mutant and the WT were found for $\mathrm{GuHCl}$ (about 5\% in both cases; Fig. 4b). The results suggest the rapid generation of $\mathrm{NH}_{4} \mathrm{Cl}$ resistant-variants in FMDV VP3 A116V + VP1 N17D populations, a feature not observed for $\mathrm{GuHCl}$ resistant-variants. When drug-resistant variants are present at low frequency in the viral population, a lower MOI should negatively affect the expression 
a

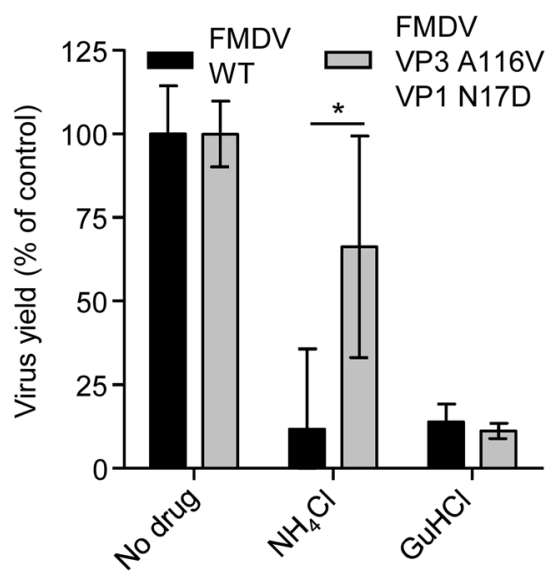

b

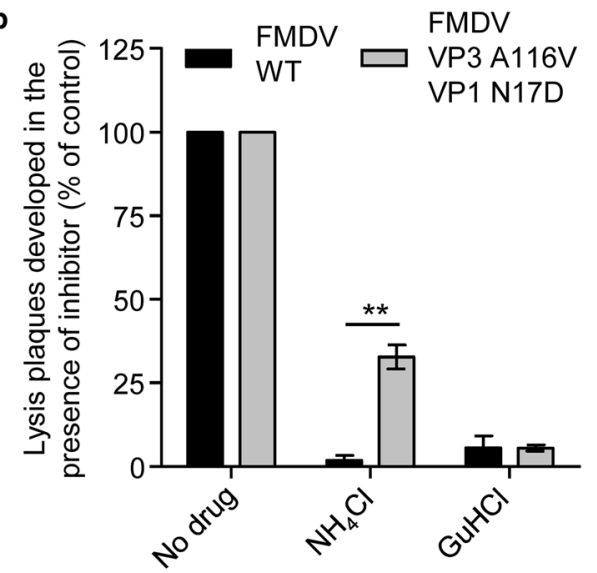

c

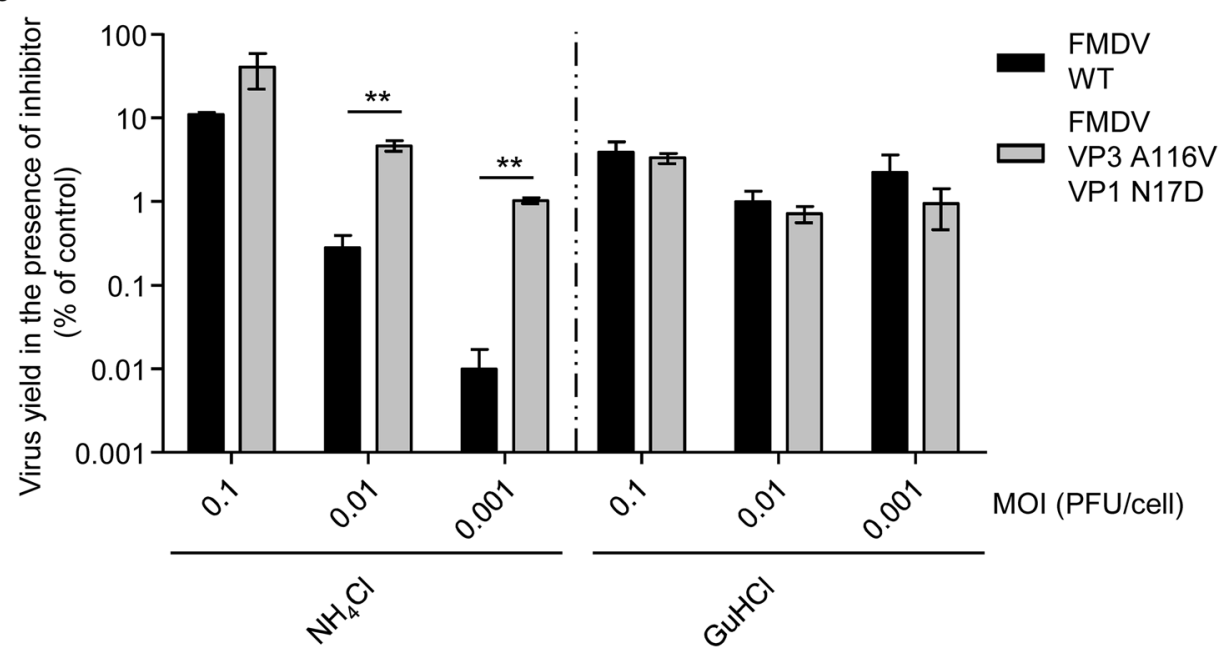

Figure 4. Viral populations carrying VP3 A116V and VP1 N17D replacements display increased resistance to $\mathrm{NH}_{4} \mathrm{Cl}$ but not to GuHCl. (a) Analysis of the resistance of FMDV WT and FMDV VP3 A116V + VP1 N17D to $\mathrm{NH}_{4} \mathrm{Cl}$ and $\mathrm{GuHCl}$. BHK-21 cells treated or not with $25 \mathrm{mM} \mathrm{NH}_{4} \mathrm{Cl}$ or $4 \mathrm{mM} \mathrm{GuHCl}$, were infected with FMDV WT or FMDV VP3 A116V VP1 N17D at a MOI of $0.5 \mathrm{PFU/cell.} \mathrm{Virus} \mathrm{yield} \mathrm{at} 8 \mathrm{~h}$ post-infection was determined by standard plaque assay. Two tailed Student's $t$-test $P$ values between control and drug-treated samples were corrected for multiple comparisons using the Sidak-Bonferroni method $\left({ }^{*} P<0.05\right)$. Data represent the means \pm SDs $(n=3-6)$. (b) Plaque assay of FMDV WT and FMDV VP3 A116V + VP1 N17D in the absence or presence of inhibitors. Viral progeny obtained from transfection of viral RNA from infectious clones was titrated in in semi-solidum medium in the absence of drug or the presence of $25 \mathrm{mM} \mathrm{NH}_{4} \mathrm{Cl}, 4 \mathrm{mM} \mathrm{GuHCl}$. The number of lysis plaques produced in each condition was determined by crystal violet staining $(30 \mathrm{~h}$ postinfection) and is expressed as the percentage of PFU developed in presence of the drug relative to that obtained in absence of the drug $(n=3-6)$. (c) Effect of $\mathrm{MOI}$ on the resistance against $\mathrm{NH}_{4} \mathrm{Cl}$ and $\mathrm{GuHCl}$. Monolayers of BHK-21 cells treated or not with $25 \mathrm{mM} \mathrm{NH}_{4} \mathrm{Cl}, 4 \mathrm{mM} \mathrm{GuHCl}$ or no inhibitor (control) were infected with FMDV WT or FMDV VP3 A116V + VP1 N17D, at a MOI of 0.1, 0.01 or $0.001 \mathrm{PFU} /$ cell. Virus yield produced in the presence of each drug $(8 \mathrm{~h}$ post-infection) was determined by standard plaque assay and is expressed as a percentage of that obtained in the absence of inhibitors (control) $(n=3)$. Two tailed Student's $t$-test $P$ values between control and drug-treated samples were corrected for multiple comparisons using the Sidak-Bonferroni method $\left({ }^{*} \mathrm{P}<0.005\right)$. Data represent the means \pm SDs.

of the phenotype $e^{40}$. The impact of reducing MOI on the degree of resistance against $\mathrm{NH}_{4} \mathrm{Cl}$ and $\mathrm{GuHCl}$ was analyzed using viral populations directly recovered from transfection of in vitro synthesized viral RNA (Fig. 4c). The results show that the ability to grow in the presence of $\mathrm{NH}_{4} \mathrm{Cl}$ was negatively affected by lowering MOI. Nevertheless, FMDV VP3 A116V + VP1 N17D was significantly less affected than WT population at all MOI tested, with the difference between these viruses being accentuated at low MOI (Fig. 4c). No effect of decreasing MOI was observed in the case of the treatment with $\mathrm{GuHCl}$. These results suggest that whereas $\mathrm{NH}_{4} \mathrm{Cl}$-resistant minority variants are present in both FMDV WT and FMDV VP3 A116V VP1 N17D populations they are more represented in the double mutant populations. 


\begin{tabular}{|c|c|c|c|c|}
\hline \multirow{3}{*}{$\begin{array}{l}\text { Proportion of genomes carrying substitutions previously associated with resistance } \\
\text { to } \mathrm{NH}_{4} \mathrm{Cl}^{\mathrm{a}}\end{array}$} & \multicolumn{4}{|l|}{ FMDV } \\
\hline & \multicolumn{2}{|l|}{ WT } & \multicolumn{2}{|c|}{$\begin{array}{l}\text { VP3 A116V+ VP1 } \\
\text { N17D }\end{array}$} \\
\hline & No drug & $\mathrm{NH}_{4} \mathrm{Cl}$ & No drug & $\mathrm{NH}_{4} \mathrm{Cl}$ \\
\hline C2895A (VP3 D115E) & 0 & $2.1 \times 10-4$ & $2.2 \times 10-4$ & $4.6 \times 10^{-4}$ \\
\hline G2896T (VP3 A116T) & 0 & 0 & 0 & 0 \\
\hline C2897T (VP3 A116V) & $2.55 \times 10^{-3}$ & $3.96 \times 10^{-3}$ & 0.99 & 0.99 \\
\hline C2903T (VP3 A118V) & 0 & 0 & 0 & 0 \\
\hline G2917A (VP3 A123T) & 0 & 0 & 0 & 0 \\
\hline G3238A (VP1 V11I) & 0 & 0 & 0 & 0 \\
\hline A3241G (VP1 T12A) & 0 & 0 & 0 & $5.2 \times 10^{-4}$ \\
\hline C3242T (VP1 T12I) & 0 & 0 & 0 & 0 \\
\hline T3259C (VP1 Y18H) & 0 & 0 & 0 & 0 \\
\hline
\end{tabular}

Table 1. Analysis of mutation frequency by NGS. ${ }^{\text {TT }}$ The average proportions of each substitution conferring $\mathrm{NH} 4 \mathrm{Cl}$ resistance ${ }^{11,13}$ were determined by NGS in three independent experiments (MOI of $0.01 \mathrm{PFU} / \mathrm{cell}$ ).

Presence of $\mathrm{NH}_{4} \mathrm{Cl}$-resistant substitutions in FMDV VP3 A116V + VP1 N17D populations. We have previously described that the pentameric interface of VP3 together with the N terminus of VP1 constitute important hot spots for the selection of mutations that modulate acid sensitivity of FMDV capsid ${ }^{11,13}$. Therefore, the proportion of genomes carrying substitutions previously related with $\mathrm{NH}_{4} \mathrm{Cl}$ resistance in this genomic region was analyzed by NGS (Table 1). For this purpose, FMDV WT and double mutant VP3 A116V + VP1 N17D were subjected or not to a single round of $\mathrm{NH}_{4} \mathrm{Cl}$ treatment. Confirming the validity of the approach, the proportion of genomes encoding VP3 A116V substitution was close to 1 in the double mutant and markedly lower in WT. These analyses indicated that two nucleotide changes (C2985T and A3241G) introducing amino acid replacements VP3 D115E and VP1 T12A previously associated with increased resistance with $\mathrm{NH}_{4} \mathrm{Cl}$ were more represented in double mutant populations treated with $\mathrm{NH}_{4} \mathrm{Cl}$ when compared to WT FMDV grown in the presence or in the absence of $\mathrm{NH}_{4} \mathrm{Cl}$. In the case of VP3 D115E, this substitution was also more represented in the double mutant populations not subjected to $\mathrm{NH}_{4} \mathrm{Cl}$ treatment (Table 1). Taken together, these results evidence/support the predisposition of mutant VP3 A116V + VP1 N17D to acquire additional mutations leading to increased $\mathrm{NH}_{4} \mathrm{Cl}$ resistance.

Fitness gain of FMDV VP3 A116V + VP1 N17D is associated with VP1 substitution T22N. Viral fitness measures the capacity to produce infectious progeny ${ }^{41,42}$. To compare the fitness of FMDV WT and FMDV VP3 A116V + VP1 N17D, viral populations directly recovered from infectious clones were tested in competition experiments performed in the absence or presence of $\mathrm{NH}_{4} \mathrm{Cl}$ (Fig. $5 \mathrm{a}, \mathrm{b}$ ). Equal PFU of each of virus populations were mixed, and used to infect cells treated or not with $\mathrm{NH}_{4} \mathrm{Cl}$. Virus recovered from the initial infection was harvested and further passaged a total of ten times either in absence or presence of $\mathrm{NH}_{4} \mathrm{Cl}$. The percentage of competing genomes during the passages was determined (Figs. $4 \mathrm{~b}, 5 \mathrm{a}$ ). In each of the three independent experiments performed in the absence of $\mathrm{NH}_{4} \mathrm{Cl}$, the genome of FMDV VP3 A116V+VP1 N17D became dominant in the second passage (about $80 \%$ of the total viral population) and its proportion progressively increased during passages up to values close to $100 \%$ (Fig. 4a). Even more pronounced was the dominance of FMDV A116V + VP1 N17D in each of three independent passage series performed in the presence of $\mathrm{NH}_{4} \mathrm{Cl}$ (about $100 \%$ of the total viral population by the second passage) and such dominance was maintained until the tenth passage (Fig. 5b). Sequencing of the capsid-coding region of the populations competing in the presence of $\mathrm{NH}_{4} \mathrm{Cl}$ showed an additional nucleotide change, C3272A, responsible for amino acid substitution T22N in VP1 that rapidly became dominant (between passage 2 and 4) in the three independent passage series (Fig. 5c). No additional mutations were found in the populations competing in the absence of $\mathrm{NH}_{4} \mathrm{Cl}$. Replacement VP1 T22N has been previously associated with an increase in acid lability of the FMDV capsid ${ }^{13}$, which further supports that the mechanism of adaptation to alkalinized endosomes in $\mathrm{NH}_{4} \mathrm{Cl}$-treated cells was based on an elevation of the $\mathrm{pH}$ threshold for uncoating. These results suggest that the double amino acid replacement A116V in VP3 and N17D in VP1 may facilitate selection of T22N in VP1, a substitution that elevates the pH threshold for uncoating.

Overall, these results show an increase in viral fitness in FMDV VP3 A116V + VP1 N17D that would enable the rapid selection of $\mathrm{NH}_{4} \mathrm{Cl}$-resistant variants, which relate to the $\mathrm{NH}_{4} \mathrm{Cl}$-resistance-prone phenotype of FMDV VP3 A116V + VP1 N17D.

\section{Discussion}

The present study has addressed the relationship between acid stability of FMDV capsid and the requirement for endosomal $\mathrm{pH}$ acidification for uncoating. We evaluated the resistance to $\mathrm{NH}_{4} \mathrm{Cl}$ of a panel of FMDV variants displaying different acid stability. $\mathrm{NH}_{4} \mathrm{Cl}$ was selected for these studies because our previous work with V-ATPase inhibitors such as concanamycin A has shown that they are less potent FMDV inhibitors than $\mathrm{NH}_{4} \mathrm{Cl}$ maybe because they block the acidification of endosomes while $\mathrm{NH}_{4} \mathrm{Cl}$ neutralizes more efficiently the endosomal $\mathrm{pH}$ by acting as a proton sink inside endosomes ${ }^{11,12}$. Unexpectedly, a genetically engineered FMDV that combined 

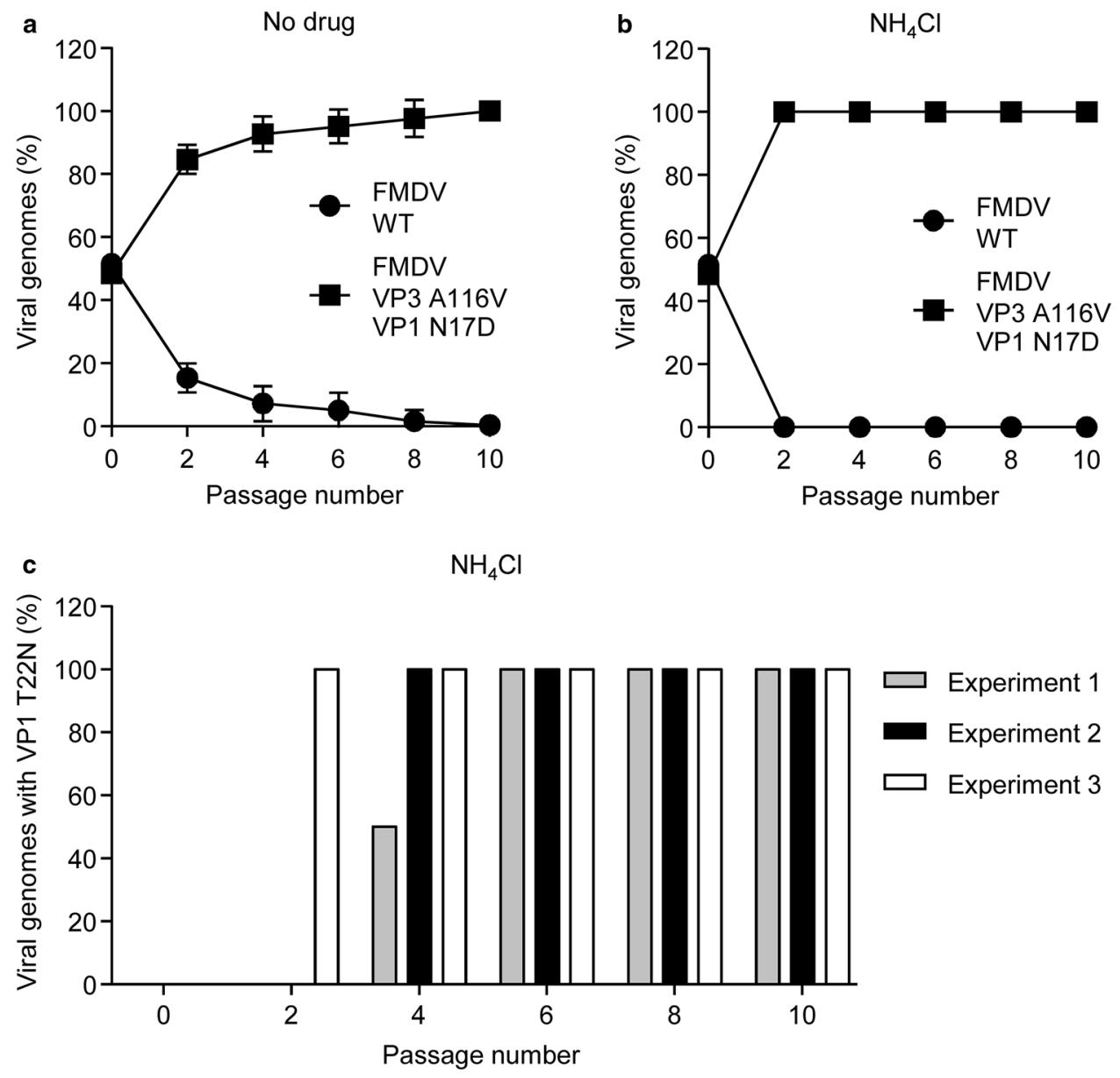

Figure 5. Fitness gain of VP3 A116V + VP1 N17D mutant. (a, b) Increase in fitness conferred by VP3 $\mathrm{A} 116 \mathrm{~V}+\mathrm{VP1} \mathrm{N} 17 \mathrm{D}$ replacements both in presence and in absence of $\mathrm{NH}_{4} \mathrm{Cl}$. Competition experiments between WT and double mutant VP3 A116V + VP1 N17D during serial passage of a virus mixture, in the absence (a) or in the presence of $25 \mathrm{mM} \mathrm{NH}_{4} \mathrm{Cl}(\mathbf{b})$. An initial MOI of $0.1 \mathrm{PFU} /$ cell (0.05 PFU/cell for each virus) was used for each infection. Approximate percentage of competing genomes during serial passages was determined by sequencing the capsid coding cDNA. Passage 0 denote the initial mixture of the viruses. Data represent the means \pm SDs $(n=3)$. (c) Rapid fixation of VP1 T22N replacement (nucleotide substitution $\mathrm{C} 3272 \mathrm{~A}$ ) in VP3 A116V + VP1 N17D mutant passaged in presence of $25 \mathrm{mM} \mathrm{NH}_{4} \mathrm{Cl}$. The percentage of genomes carrying nucleotide substitution $\mathrm{C} 3272 \mathrm{~A}$ was calculated in three independent passage series (Experiments 1, 2 and 3).

amino acid replacements $\mathrm{A} 116 \mathrm{~V}$ in VP3 and N17D in VP1 exhibited similar acid resistance than the parental FMDV ${ }^{13}$ but displayed increased resistance to $\mathrm{NH}_{4} \mathrm{Cl}$. This mutant virus combined amino acid replacement VP3 A116V, which confers increased acid lability and hence induces resistance to $\mathrm{NH}_{4} \mathrm{Cl}^{11,13}$, with amino acid replacement VP1 N17D, which provides increased acid stability and hence increases sensitivity to $\mathrm{NH}_{4} \mathrm{Cl}^{12,17,19}$. Interestingly, whereas the combination of both substitutions in a single genome resulted in an additive effect and led to a virus with similar acid sensitivity than WT, this variant showed higher resistance to $\mathrm{NH}_{4} \mathrm{Cl}$.

The escape from $\mathrm{NH}_{4} \mathrm{Cl}$ is concomitant with a rapid increase in the acid lability of the FMDV VP3 A116V + VP1 N17D population. This change in acid lability could not initially be explained by the selection of a single or a reduced number of mutations in the consensus sequence, suggesting that the mutant cloud composition may be responsible for this phenotypic change. Such effects on fitness recovery have been previously observed for other viral models ${ }^{43}$. Our experiments to analyze the frequency of $\mathrm{NH}_{4} \mathrm{Cl}$-resistant variants and the effect of decreasing MOI on resistance to $\mathrm{NH}_{4} \mathrm{Cl}$ also supported that although both FMDV WT and FMDV VP3 $\mathrm{A} 116 \mathrm{~V}+\mathrm{VP} 1 \mathrm{~N} 17 \mathrm{D}$ populations showed $\mathrm{NH}_{4} \mathrm{Cl}$-resistant minority variants, they were 18 -fold more represented in the FMDV VP3 A116V + VP1 N17D population. Therefore, it seemed that the presence of the two substitutions VP3 A116V and VP1 N17D might facilitate selection of $\mathrm{NH}_{4} \mathrm{Cl}$-resistant variants. NGS analyses confirmed/ supported this hypothesis for two different nucleotide replacements leading to amino acid substitutions (VP3 D115E and VP1 T12A) previously related to $\mathrm{NH}_{4} \mathrm{Cl}$ resistance. Further passaging in the presence of the $\mathrm{NH}_{4} \mathrm{Cl}$ during competition experiments led to the fixation of an additional amino acid replacement (VP1 T22N) located close to D17 in the N-terminal region of $\mathrm{VP} 1^{44}$ previously linked to increased acid lability of FMDV capsid, and resistance to $\mathrm{NH}_{4} \mathrm{Cl}^{13}$. These observations suggest that during the treatment with $\mathrm{NH}_{4} \mathrm{Cl}$, variants such as those 
with VP3 D115E, VP1 T12A, VP1 T22N may be present at frequencies insufficient to modify the consensus sequence but that can be readily selected by $\mathrm{NH}_{4} \mathrm{Cl}$.

Our previous work has shown a variety of amino acid substitutions in the FMDV capsid as responsible for $\mathrm{NH}_{4} \mathrm{Cl}$ resistance ${ }^{11,13}$. However, in the three competition experiments between FMDV WT and FMDV VP3 A116V + VP1 N17D, the same amino acid replacement (VP1 T22N) was independently selected. The selection of this amino acid replacement was also previously documented during serial passage of this double mutant in $\mathrm{NH}_{4} \mathrm{Cl}$ in an independent series of experiments ${ }^{13}$. Thus, the tendency for the selection of replacement VP1 T22N points to deterministic constrains for capsid evolution in the presence of $\mathrm{NH}_{4} \mathrm{Cl}$, probably explained by a selective advantage of VP1 T22N in the context of the VP1 N17D and VP3 A116V double substitution. Regarding the increase in acid resistance as a result of the introduction of VP1 T22N, it was previously proposed that the complexity of the interactions established by the residues located at the N terminus of VP1 with multiple residues located in VP4 and also in VP2 and VP3 could modulate acid-resistance ${ }^{13}$. However, further work should be performed to elucidate the molecular mechanism behind the increase in acid sensitivity induced by this replacement. The results of the competition experiments indicated also that the double mutant exhibited an increase in biological fitness in both the presence and the absence of $\mathrm{NH}_{4} \mathrm{Cl}$, although it was more obvious in the presence of $\mathrm{NH}_{4} \mathrm{Cl}$. The lack of resistance of the double mutant to $\mathrm{GuHCl}$, an inhibitor of replication that targets the viral polymerase, indicates that fitness per se, as a multidrug resistance trait documented for hepatitis $\mathrm{C}$ virus ${ }^{40}$, is not the mechanism of $\mathrm{NH}_{4} \mathrm{Cl}$ resistance in FMDV VP3 A116V + VP1 N17D. High fitness may facilitate explorations of sequence space to select for substitution VP1 T22N that expresses the resistance phenotype in the context of the double substitution. There is previous evidence that VP1 N17D replacement in its own did not alter biological fitness of FMDV C-S8c1 at neutral $\mathrm{pH}^{12}$, nor was it associated with a loss of fitness at neutral $\mathrm{pH}$ in another FMDV serotype ${ }^{19}$. However, mutants carrying VP1 N17D together with an additional substitution (H145Y) displayed an increase in viral fitness at neutral $\mathrm{pH}^{17}$. This result is in agreement with other evolutionary studies showing that although pathogens that develop resistance to drugs usually have reduced fitness, compensatory mutations that restore fitness may facilitate the stability of the resistance phenotype $\mathrm{e}^{45,46}$.

Viral capsids are metastable assemblies evolved to protect the genome while allowing its release for infection ${ }^{1}$. Upon acidification, the FMDV capsid undergoes a profound and irreversible conformational alteration that leads to its dissociation into pentameric subunits. We have previously demonstrated that other amino acid substitutions similar to VP3 A116V, such as VP3 A118V, facilitate capsid dissociation through the introduction of bulkier amino acid side chains close to the pentameric interfaces, thus debilitating interpentameric interactions ${ }^{11}$. However, this effect was counteracted by amino acid substitutions that stabilize the capsid ${ }^{13}$. In this way, a balance between stability and dissociability should be conserved by both WT and double mutant FMDV VP3 A116V + VP1 N17D. Our data suggest that this double mutant could displace this balance towards the generation of acid-labile populations more easily than FMDV WT. Therefore, the fitness increase of the double mutant together with a lower threshold for destabilization could explain the increased ability to produce acid-labile capsid of the double mutant population and hence to escape from the inhibitory effect of $\mathrm{NH}_{4} \mathrm{Cl}$. From an evolutionary perspective, the results show how the presence of specific combination of mutations in a viral genome can modify phenotypes in unpredictable manners and the capacity of a virus to respond to a selective constraint.

In summary, the present study provides novel evidences showing that the combination of mutations with opposite effects on acid-stability can result in compensatory effects that can lead to an unforeseen fitness gain, and facilitate rapid adaptation against an inhibitor of acid-dependent uncoating.

Received: 12 May 2021; Accepted: 15 November 2021

Published online: 06 December 2021

\section{References}

1. Mateu, M. G. Assembly, stability and dynamics of virus capsids. Arch. Biochem. Biophys. 531, 65-79. https://doi.org/10.1016/j.abb. 2012.10.015 (2013).

2. Acharya, R. et al. The three-dimensional structure of foot-and-mouth disease virus at 2.9 A resolution. Nature 337, 709-716 (1989).

3. Newman, J. F., Rowlands, D. J. \& Brown, F. A physico-chemical sub-grouping of the mammalian picornaviruses. J. Gen. Virol. 18, $171-180(1973)$.

4. Grubman, M. J. \& Baxt, B. Foot-and-mouth disease. Clin. Microbiol. Rev. 17, 465-493 (2004).

5. Vazquez-Calvo, A., Saiz, J. C., McCullough, K. C., Sobrino, F. \& Martin-Acebes, M. A. Acid-dependent viral entry. Virus Res. 20, 20 (2012).

6. Vazquez-Calvo, A. et al. Modulation of foot-and-mouth disease virus $\mathrm{pH}$ threshold for uncoating correlates with differential sensitivity to inhibition of cellular Rab GTPases and decreases infectivity in vivo. J. Gen. Virol. 93, 2382-2386. https://doi.org/10. 1099/vir.0.045419-0 (2012).

7. Yuan, H. et al. The $\mathrm{pH}$ stability of foot-and-mouth disease virus. Virol. J. 14, 233. https://doi.org/10.1186/s12985-017-0897-z (2017).

8. van Vlijmen, H. W., Curry, S., Schaefer, M. \& Karplus, M. Titration calculations of foot-and-mouth disease virus capsids and their stabilities as a function of pH. J. Mol. Biol. 275, 295-308 (1998).

9. Baxt, B. Effect of lysosomotropic compounds on early events in foot-and-mouth disease virus replication. Virus Res. 7, 257-271 (1987).

10. Martin-Acebes, M. A., Gonzalez-Magaldi, M., Sandvig, K., Sobrino, F. \& Armas-Portela, R. Productive entry of type C footand-mouth disease virus into susceptible cultured cells requires clathrin and is dependent on the presence of plasma membrane cholesterol. Virology 369, 105-118. https://doi.org/10.1016/j.virol.2007.07.021 (2007).

11. Martin-Acebes, M. A., Rincon, V., Armas-Portela, R., Mateu, M. G. \& Sobrino, F. A single amino acid substitution in the capsid of foot-and-mouth disease virus can increase acid lability and confer resistance to acid-dependent uncoating inhibition. J. Virol. 84, 2902-2912 (2010).

12. Martin-Acebes, M. A., Vazquez-Calvo, A., Rincon, V., Mateu, M. G. \& Sobrino, F. A single amino acid substitution in the capsid of foot-and-mouth disease virus can increase acid resistance. J. Virol. 85, 2733-2740. https://doi.org/10.1128/JVI.02245-10 (2011). 
13. Caridi, F., Vazquez-Calvo, A., Sobrino, F. \& Martin-Acebes, M. A. The pH stability of foot-and-mouth disease virus particles is modulated by residues located at the pentameric interface and in the N terminus of VP1. J. Virol. 89, 5633-5642. https://doi.org/ 10.1128/JVI.03358-14 (2015).

14. Domingo, E. et al. Evolution of foot-and-mouth disease virus. Virus Res. 91, 47-63 (2003).

15. Twomey, T. et al. Characterization of an acid-resistant mutant of foot-and-mouth disease virus. Virology 206, 69-75 (1995).

16. Biswal, J. K., Das, B., Sharma, G. K., Khulape, S. A. \& Pattnaik, B. Role of a single amino acid substitution of VP3 H142D for increased acid resistance of foot-and-mouth disease virus serotype A. Virus Genes 52, 235-243. https://doi.org/10.1007/s11262016-1294-1 (2016).

17. Wang, H. et al. Single amino acid substitution of VP1 N17D or VP2 H145Y confers acid-resistant phenotype of type Asial footand-mouth disease virus. Virol. Sin. 29, 103-111. https://doi.org/10.1007/s12250-014-3426-x (2014).

18. Vazquez-Calvo, A., Caridi, F., Sobrino, F. \& Martin-Acebes, M. A. An increase in acid resistance of foot-and-mouth disease virus capsid is mediated by a tyrosine replacement of the VP2 histidine previously associated with VP0 cleavage. J. Virol. 88, 3039-3042. https://doi.org/10.1128/JVI.03222-13 (2014).

19. Liang, T. et al. Selection and characterization of an acid-resistant mutant of serotype $\mathrm{O}$ foot-and-mouth disease virus. Adv. Virol. 159, 657-667. https://doi.org/10.1007/s00705-013-1872-7 (2014).

20. Bai, X. W. et al. Engineering responses to amino acid substitutions in the VP0- and VP3-coding regions of panasia-1 strains of foot-and-mouth disease virus serotype O. J. Virol. https://doi.org/10.1128/JVI.02278-18 (2019).

21. Gebauer, F. et al. Rapid selection of genetic and antigenic variants of foot-and-mouth disease virus during persistence in cattle. J. Virol. 62, 2041-2049 (1988).

22. Dopazo, J., Sobrino, F., Palma, E. L., Domingo, E. \& Moya, A. Gene encoding capsid protein VP1 of foot-and-mouth disease virus: A quasispecies model of molecular evolution. Proc. Natl. Acad. Sci. USA 85, 6811-6815 (1988).

23. Martinez, M. A., Verdaguer, N., Mateu, M. G. \& Domingo, E. Evolution subverting essentiality: Dispensability of the cell attachment Arg-Gly-Asp motif in multiply passaged foot-and-mouth disease virus. Proc. Natl. Acad. Sci. USA 94, 6798-6802. https:// doi.org/10.1073/pnas.94.13.6798 (1997).

24. Luna, E., Rodriguez-Huete, A., Rincon, V., Mateo, R. \& Mateu, M. G. Systematic study of the genetic response of a variable virus to the introduction of deleterious mutations in a functional capsid region. J. Virol. 83, 10140-10151. https://doi.org/10.1128/JVI. 00903-09 (2009).

25. Mateo, R. \& Mateu, M. G. Deterministic, compensatory mutational events in the capsid of foot-and-mouth disease virus in response to the introduction of mutations found in viruses from persistent infections. J. Virol. 81, 1879-1887 (2007).

26. Mateu, M. G. Antibody recognition of picornaviruses and escape from neutralization: A structural view. Virus Res. 38, 1-24 (1995).

27. Fares, M. A. et al. Evidence for positive selection in the capsid protein-coding region of the foot-and-mouth disease virus (FMDV) subjected to experimental passage regimens. Mol. Biol. Evol. 18, 10-21. https://doi.org/10.1093/oxfordjournals.molbev.a003715 (2001).

28. Tully, D. C. \& Fares, M. A. Shifts in the selection-drift balance drive the evolution and epidemiology of foot-and-mouth disease virus. J. Virol. 83, 781-790. https://doi.org/10.1128/JVI.01500-08 (2009).

29. Escarmis, C., Perales, C. \& Domingo, E. Biological effect of Muller's Ratchet: Distant capsid site can affect picornavirus protein processing. J. Virol. 83, 6748-6756. https://doi.org/10.1128/JVI.00538-09 (2009).

30. Mateo, R., Díaz, A., Baranowski, E. \& Mateu, M. G. Complete alanine scanning of intersubunit interfaces in a foot-and-mouth disease virus capsid reveals critical contributions of many side chains to particle stability and viral function. J. Biol. Chem. 278, 41019-41027 (2003).

31. Lopez-Arguello, S. et al. Thermostability of the foot-and-mouth disease virus capsid is modulated by lethal and viability-restoring compensatory amino acid substitutions. J. Virol. https://doi.org/10.1128/JVI.02293-18 (2019).

32. Garcia-Arriaza, J., Manrubia, S. C., Toja, M., Domingo, E. \& Escarmis, C. Evolutionary transition toward defective RNAs that are infectious by complementation. J. Virol. 78, 11678-11685. https://doi.org/10.1128/JVI.78.21.11678-11685.2004 (2004).

33. Knipe, T., Rieder, E., Baxt, B., Ward, G. \& Mason, P. W. Characterization of synthetic foot-and-mouth disease virus provirions separates acid-mediated disassembly from infectivity. J. Virol. 71, 2851-2856 (1997).

34. Perales, C., Agudo, R., Tejero, H., Manrubia, S. C. \& Domingo, E. Potential benefits of sequential inhibitor-mutagen treatments of RNA virus infections. PLoS Pathog. 5, e1000658. https://doi.org/10.1371/journal.ppat.1000658 (2009).

35. Caridi, F. et al. Negatively charged amino acids at the foot-and-mouth disease virus capsid reduce the virion-destabilizing effect of viral RNA at acidic pH. Sci. Rep. https://doi.org/10.1038/s41598-020-58414-8 (2020).

36. Caridi, F., Cañas-Arranz, R., Vazquez-Calvo, A., Sobrino, F. \& Martin-Acebes, M. A. Equine Rhinitis A virus mutants with altered acid resistance unveil a key role of VP3 and intrasubunit interactions in the control of the $\mathrm{pH}$ stability of the aphthovirus capsid. J. Virol. 90, 25 (2016).

37. Rincon, V. et al. Identification of the structural basis of thermal lability of a virus provides a rationale for improved vaccines. Structure 22, 25 (2014).

38. Saunders, K. \& King, A. M. Guanidine-resistant mutants of aphthovirus induce the synthesis of an altered nonstructural polypeptide, P34. J. Virol. 42, 389-394. https://doi.org/10.1128/JVI.42.2.389-394.1982 (1982).

39. Pariente, N., Airaksinen, A. \& Domingo, E. Mutagenesis versus inhibition in the efficiency of extinction of foot-and-mouth disease virus. J. Virol. 77, 7131-7138. https://doi.org/10.1128/jvi.77.12.7131-7138.2003 (2003).

40. Sheldon, J. et al. Increased replicative fitness can lead to decreased drug sensitivity of hepatitis C virus. J. Virol. 88, 12098-12111. https://doi.org/10.1128/JVI.01860-14 (2014).

41. Domingo, E., Sheldon, J. \& Perales, C. Viral quasispecies evolution. Microbiol. Mol. Biol. Rev. 76, 159-216. https://doi.org/10.1128/ MMBR.05023-11 (2012).

42. Domingo, E., de Avila, A. I., Gallego, I., Sheldon, J. \& Perales, C. Viral fitness: History and relevance for viral pathogenesis and antiviral interventions. Pathog. Dis. https://doi.org/10.1093/femspd/ftz021 (2019).

43. Borderia, A. V. et al. Initial fitness recovery of HIV-1 is associated with quasispecies heterogeneity and can occur without modifications in the consensus sequence. PLoS One 5, e10319. https://doi.org/10.1371/journal.pone.0010319 (2010).

44. Lea, S. et al. The structure and antigenicity of a type C foot-and-mouth disease virus. Structure 2, 123-139. https://doi.org/10. 1016/s0969-2126(00)00014-9 (1994).

45. Handel, A., Regoes, R. R. \& Antia, R. The role of compensatory mutations in the emergence of drug resistance. PLoS Comput. Biol. 2, e137. https://doi.org/10.1371/journal.pcbi.0020137 (2006).

46. Bottger, E. C., Springer, B., Pletschette, M. \& Sander, P. Fitness of antibiotic-resistant microorganisms and compensatory mutations. Nat. Med. 4, 1343-1344. https://doi.org/10.1038/3906 (1998).

\section{Acknowledgements}

This work was funded by Comunidad de Madrid co-financed with ECFEDER funds (P2018/BAA-4370). Work in F.S.'s laboratory was also funded by Grants from MINECO/MICINN-FEDER EU (AGL2017-84097C2-1-R; PID2020-113184RB-C21), and through the Spanish National Reserarch Council (CSIC), grant CONEXIONES-Vida/202120E47. Work in E.D.'s laboratory was also funded by MICIN/-FEDER EU grant 
PID2020-113888RB-100. Work in CBMSO groups was also funded by an institutional grant from Fundación Ramón Areces. We thank R. Peiró-Pastor for his aid in sequencing analyses.

\section{Author contributions}

F.C., R.C.-A., A.V.-C., P.L. and K.I.C. performed the experiments in figures 1-4. F.C., E.D., F.S. and M.A.M-A wrote the main manuscript text. F.C. and M.A.M-A prepared figures 1-5 and Table 1. All authors reviewed the manuscript.

\section{Funding}

This article was funded by MINECO-FEDER EU (Grant no. AGL2017-84097-C2-1-R), Comunidad de Madrid (Grant no. P2018/BAA-4370) and Fundación Ramón Areces.

\section{Competing interests}

The authors declare no competing interests.

\section{Additional information}

Correspondence and requests for materials should be addressed to F.S.

Reprints and permissions information is available at www.nature.com/reprints.

Publisher's note Springer Nature remains neutral with regard to jurisdictional claims in published maps and institutional affiliations.

(c) (i) Open Access This article is licensed under a Creative Commons Attribution 4.0 International License, which permits use, sharing, adaptation, distribution and reproduction in any medium or format, as long as you give appropriate credit to the original author(s) and the source, provide a link to the Creative Commons licence, and indicate if changes were made. The images or other third party material in this article are included in the article's Creative Commons licence, unless indicated otherwise in a credit line to the material. If material is not included in the article's Creative Commons licence and your intended use is not permitted by statutory regulation or exceeds the permitted use, you will need to obtain permission directly from the copyright holder. To view a copy of this licence, visit http://creativecommons.org/licenses/by/4.0/.

(C) The Author(s) 2021 